PROCEEDINGS OF THE

AMERICAN MATHEMATICAL SOCIETY

Volume 133, Number 9, Pages 2549-2557

S 0002-9939(05)07290-4

Article electronically published on April 12, 2005

\title{
UNIQUENESS OF POSITIVE SOLUTIONS FOR SINGULAR PROBLEMS INVOLVING THE $p$-LAPLACIAN
}

\author{
ARKADY POLIAKOVSKY AND ITAI SHAFRIR
}

(Communicated by David S. Tartakoff)

\begin{abstract}
We study existence and uniqueness of positive eigenfunctions for the singular eigenvalue problem: $-\Delta_{p} u-\lambda \eta(x) \frac{u^{p-1}}{|x|^{p}}=\mu \frac{u^{p-1}}{|x|^{p}}$ on a bounded smooth domain $\Omega \subset \mathbb{R}^{N}$ with zero boundary condition. We also characterize all positive solutions of $-\Delta_{p} u=\left|\frac{N-p}{p}\right|^{p} \frac{u^{p-1}}{|x|^{p}}$ in $\mathbb{R}^{N} \backslash\{0\}$.
\end{abstract}

\section{INTRODUCTION}

This note is concerned with solutions of the following nonlinear eigenvalue problem:

$$
\left\{\begin{array}{lll}
-\Delta_{p} u-\lambda \eta(x) \frac{|u|^{p-2} u}{|x|^{p}} & =\mu \frac{|u|^{p-2} u}{|x|^{p}} \quad \text { in } \Omega \backslash\{0\}, \\
u & >0 \text { in } \Omega \backslash\{0\}, \\
u & =0 \text { on } \partial \Omega .
\end{array}\right.
$$

Here $\Omega$ is a bounded domain in $\mathbb{R}^{N}$ of class $C^{2}$ with $0 \in \Omega, p \in(1, \infty) \backslash\{N\}$, $\eta \in C^{\alpha}(\bar{\Omega})(\alpha \in(0,1))$ such that $\eta \geq 0, \eta \not \equiv 0$ in $\bar{\Omega}$ and $\eta(0)=0, \mu, \lambda \in \mathbb{R}$ are two parameters and $\Delta_{p} u=\operatorname{div}\left(|\nabla u|^{p-2} \nabla u\right)$. By the regularity results of Tolksdorf [11] and Di Benedetto [4] any weak solution $u \in \bigcap_{r>0} W^{1, p}\left(\Omega \backslash B_{r}(0)\right)$ to (1.1) actually belongs to $C^{1}(\bar{\Omega} \backslash\{0\})$.

Naturally related to (1.1) is the following variational problem:

$$
I[\lambda]:=\inf _{0 \neq v \in W_{0}^{1, p}(\Omega \backslash\{0\})} \frac{\int_{\Omega}|\nabla v|^{p}-\lambda \int_{\Omega} \frac{\eta|v|^{p}}{|x|^{p}}}{\int_{\Omega}(|v| /|x|)^{p}} .
$$

Recall that in the case $\lambda=0$ it follows from the well-known Hardy inequality,

$$
\int_{\Omega}|\nabla u|^{p} \geq\left|\frac{N-p}{p}\right|^{p} \int_{\Omega}(|u| /|x|)^{p}, \quad \forall u \in W_{0}^{1, p}(\Omega \backslash\{0\}),
$$

that $I[0]=c_{p, N}^{*}:=\left|\frac{N-p}{p}\right|^{p}$. Moreover, by a simple construction of test functions approximating $|x|^{1-N / p}$ (see [8]), it can be shown that we always have $I[\lambda] \leq c_{p, N}^{*}$. It was also proved in [8] (in the spirit of [3]) that there exists $\lambda^{*}>0$ such that $I[\lambda]=c_{p, N}^{*}$ for $\lambda \leq \lambda^{*}$ and $I[\lambda]<c_{p, N}^{*}$ for $\lambda>\lambda^{*}$. If $u \in W_{0}^{1, p}(\Omega \backslash\{0\})$ is a minimizer for (1.2), then $u$ is clearly a solution to the equation in (1.1) with

Received by the editors March 2, 2002

2000 Mathematics Subject Classification. Primary 35J70; Secondary 49R50.

(C)2005 American Mathematical Society Reverts to public domain 28 years from publication 
$\mu=I[\lambda]$. Since $|u|$ is also a minimizer, hence a solution to the same equation, it follows from Serrin's Harnack principle [9] that $|u|>0$ in $\Omega \backslash\{0\}$. Therefore, either $u$ or $-u$ is a solution to (1.1). It turns out that solutions of (1.1) can be obtained as minimizers for (1.2) if and only if $I[\lambda]<c_{p, N}^{*}$. In this direction we have:

Theorem 1.1. A solution $u$ to (1.1) with $\mu=I[\lambda]$ which belongs to $W_{0}^{1, p}(\Omega \backslash\{0\})$ exists if and only if $I[\lambda]<c_{p, N}^{*}$. In this case $u$ is a minimizer for (1.2) and it is the unique solution to (1.1) up to a multiplicative factor.

The critical role played by the value $c_{p, N}^{*}$ for the existence of a minimizer is analogous to a phenomenon that was observed first by Marcus, Mizel and Pinchover [6] in the context of another version of Hardy's inequality. The existence of a minimizer in the subcritical case $I[\lambda]<c_{p, N}^{*}$ is proved in the appendix, using a "concentration-compactness principle" [5], similar to the one used in [6] 7]. The uniqueness part in the subcritical case is then essentially known ([2, 1, 7]); see the appendix for details.

The next natural question is what happens in the critical case $I[\lambda]=c_{p, N}^{*}$. In that case the existence of a solution $u \in \bigcap_{q \in[1, p)} W_{0}^{1, q}(\Omega \backslash\{0\}) \backslash W_{0}^{1, p}(\Omega \backslash\{0\})$ was proved in 8 . We are then left with the problem of uniqueness. This is the subject of the main theorem of this note:

Theorem 1.2. In the case $I[\lambda]=c_{p, N}^{*}$ the solution to (1.1) with $\mu=c_{p, N}^{*}$ is unique up to a multiplicative factor.

Uniqueness in the linear case $p=2$ is known and easy (for any value of $\mu$, see Remark 3.1), but we could not find an easy proof for general $p$. Our argument, presented in Section 3, is based on Harnack's inequality and on the following Liouville type theorem, which may be of independent interest.

Theorem 1.3. Let $u$ be a solution of

$$
\begin{cases}-\Delta_{p} u & =c_{p, N}^{*} \frac{u^{p-1}}{\mid x^{p}} \quad \text { in } \mathbb{R}^{N} \backslash\{0\}, \\ u & >0 \text { in } \mathbb{R}^{N} \backslash\{0\} .\end{cases}
$$

Then $u(x)=C|x|^{1-N / p}$ for some positive constant $C$.

\section{Characterization of global solutions}

In the proof of Theorem 1.3 we will make use of an extension of Picone's identity for the $p$-Laplacian, due to Allegretto and Huang [1], that we now recall. Let $\Omega$ be a subdomain of $\mathbb{R}^{N}$. For $u, v$ in $C^{1}(\Omega)$ such that $u \geq 0$ and $v>0$ in $\Omega$, denote

$$
\begin{array}{r}
L(u, v)=|\nabla u|^{p}+(p-1) \frac{u^{p}}{v^{p}}|\nabla v|^{p}-p \frac{u^{p-1}}{v^{p-1}} \nabla u|\nabla v|^{p-2} \nabla v, \\
R(u, v)=|\nabla u|^{p}-\nabla\left(\frac{u^{p}}{v^{p-1}}\right)|\nabla v|^{p-2} \nabla v .
\end{array}
$$

Then $L(u, v)=R(u, v) \geq 0$ in $\Omega$ and $L(u, v)=R(u, v)=0$ in $\Omega$ if and only if $u=C v$ for some constant $C$; see [1].

Proof of Theorem [1.3. By the regularity results of Tolksdorf [1] and Di Benedetto [4] any weak solution to (1.4) belongs to $C^{1}\left(\mathbb{R}^{N} \backslash\{0\}\right)$. Clearly $v(x)=|x|^{1-N / p}$ is 
a solution of (1.4). We must show that $u$ is a multiple of $v$. For each $R>0$ we define a cut-off function $\psi_{R}$ as follows:

$$
\psi_{R}(x)= \begin{cases}0, & 0 \leq|x| \leq \frac{1}{R^{2}}, \\ 2+\frac{\log |x|}{\log R}, & \frac{1}{R^{2}} \leq|x| \leq \frac{1}{R} \\ 1, & \frac{1}{R} \leq|x| \leq R \\ 2-\frac{\log |x|}{\log R}, & R \leq|x| \leq R^{2} \\ 0, & |x| \geq R^{2} .\end{cases}
$$

Clearly $\psi_{R}(x)$ is a Lipschitz function with compact support in $\mathbb{R}^{N}$. By Picone's identity (2.1) and (1.4),

$$
\begin{aligned}
0 \leq \int_{\mathbb{R}^{N}} L\left(\psi_{R} v, u\right) & =\int_{\mathbb{R}^{N}} R\left(\psi_{R} v, u\right)=\int_{\mathbb{R}^{N}}\left|\nabla\left(\psi_{R} v\right)\right|^{p}-\int_{\mathbb{R}^{N}} \nabla\left(\frac{\psi_{R}^{p} v^{p}}{u^{p-1}}\right)|\nabla u|^{p-2} \nabla u \\
& =\int_{\mathbb{R}^{N}}\left(\left|\nabla\left(\psi_{R} v\right)\right|^{p}-\frac{c_{p, N}^{*}}{|x|^{p}} \psi_{R}^{p} v^{p}\right) .
\end{aligned}
$$

On the other hand, testing (1.4) for $v$ against $\psi_{R}^{p} v$ yields

$$
\int_{\mathbb{R}^{N}} \frac{c_{p, N}^{*}}{|x|^{p}} \psi_{R}^{p} v^{p}=\int_{\mathbb{R}^{N}} \psi_{R}^{p}|\nabla v|^{p}+p \psi_{R}^{p-1} v|\nabla v|^{p-2} \nabla v \nabla \psi_{R} .
$$

Combining (2.3) with (2.4) we obtain,

$$
\begin{aligned}
0 & \leq I(R):=\int_{R^{N}} L\left(\psi_{R} v, u\right) \\
& =\int_{\mathbb{R}^{N}}\left(\left|\psi_{R} \nabla v+v \nabla \psi_{R}\right|^{p}-\psi_{R}^{p}|\nabla v|^{p}-p \psi_{R}^{p-1} v|\nabla v|^{p-2} \nabla v \nabla \psi_{R}\right) .
\end{aligned}
$$

Next we shall prove that $\lim _{R \rightarrow \infty} I(R)=0$. We recall the following elementary inequality which holds for all $z_{1}, z_{2} \in \mathbb{R}^{N}$ (see [10, Lemma A.4]):

$$
\left|z_{1}+z_{2}\right|^{p}-\left|z_{1}\right|^{p}-p\left|z_{1}\right|^{p-2} z_{1} \cdot z_{2} \leq \begin{cases}\frac{p(p-1)}{2}\left(\left|z_{1}\right|+\left|z_{2}\right|\right)^{p-2}\left|z_{2}\right|^{2}, & \text { for } p>2 \\ \gamma_{p}\left|z_{2}\right|^{p} & \text { for } p \leq 2\end{cases}
$$

for some constant $\gamma_{p}>0$. Assume first that $p \leq 2$. Then from (2.2), (2.5) and (2.6) we get

$$
\begin{aligned}
I(R) & \leq C\left(\int_{\frac{1}{R^{2}}}^{\frac{1}{R}} r^{p-N}\left(\frac{1}{r \log R}\right)^{p} r^{N-1} d r+\int_{R}^{R^{2}} r^{p-N}\left(\frac{1}{r \log R}\right)^{p} r^{N-1} d r\right) \\
& =\frac{2 C}{(\log R)^{p-1}},
\end{aligned}
$$

hence $\lim _{r \rightarrow \infty} I(R)=0$ in this case. Now consider the case $p>2$. Thanks to (2.6) and (2.7) we need only show that

$$
\int_{A_{R}}|\nabla v|^{p-2} v^{2}\left|\nabla \psi_{R}\right|^{2} \rightarrow 0 \text { as } R \rightarrow \infty,
$$


where $A(R)=\left\{\frac{1}{R^{2}}<|x|<\frac{1}{R}\right\} \cup\left\{R<|x|<R^{2}\right\}$. Indeed, (2.8) follows from

$$
\begin{aligned}
\int_{A_{R}}|\nabla v|^{p-2} v^{2}\left|\nabla \psi_{R}\right|^{2} \leq & C \int_{\frac{1}{R^{2}}}^{\frac{1}{R}} r^{-\frac{N}{p}(p-2)} r^{2\left(1-\frac{N}{p}\right)} \frac{r^{N-1} d r}{r^{2}(\log R)^{2}} \\
& +C \int_{R}^{R^{2}} r^{-\frac{N}{p}(p-2)} r^{2\left(1-\frac{N}{p}\right)} \frac{r^{N-1} d r}{r^{2}(\log R)^{2}} \\
& =\frac{2 C}{\log R} \rightarrow 0 \text { as } R \rightarrow \infty .
\end{aligned}
$$

Therefore, in all cases we have $\lim _{R \rightarrow \infty} \int_{R^{N}} L\left(\psi_{R} v, u\right)=0$. By Fatou's Lemma we infer that $L(v, u) \in L^{1}\left(\mathbb{R}^{N}\right)$ and $\int_{\mathbb{R}^{N}} L(v, u)=0$. Hence, $L(v, u)=0$ in $\mathbb{R}^{N}$ which implies that for some constant $C>0$ we have $u=C v=C|x|^{1-N / p}$.

From Theorem 1.3 we next deduce the asymptotic radial symmetry at the origin of solutions on a bounded domain.

Corollary 2.1. Let $u \in W_{l o c}^{1, p}\left(B_{R}(0) \backslash\{0\}\right)$ be a solution of

$$
\begin{cases}-\Delta_{p} u & =\frac{u^{p-1}}{|x|^{p}}\left(c_{p, N}^{*}+\eta(x)\right) \text { on } B_{R}(0) \backslash\{0\}, \\ u & >0 \text { on } B_{R}(0) \backslash\{0\},\end{cases}
$$

where $\eta(x) \in C\left(B_{R}(0)\right)$ satisfies $\eta(0)=0$. Then,

$$
\lim _{r \rightarrow 0^{+}} \frac{\bar{u}(r)}{\underline{u}(r)}=1
$$

where for each $r \in(0, R)$ we denote

$$
\bar{u}(r)=\max _{\{|x|=r\}} u(x), \underline{u}(r)=\min _{\{|x|=r\}} u(x) .
$$

Proof. Fix $n \in \mathbb{R}^{N}$ with $|n|=1$ and for any $r \in(0, R / 2)$ set

$$
v_{r}(y)=\frac{u(r y)}{u(r n)}, \quad \text { for } y \in B_{R / r}(0) \backslash\{0\} .
$$

Then $v_{r}$ satisfies

$$
-\Delta_{p} v_{r}=\frac{v_{r}^{p-1}}{|y|^{p}}\left(c_{p, N}^{*}+\eta(r y)\right) \quad \text { on } B_{R / r}(0) \backslash\{0\},
$$

and $v_{r}(n)=1$. For any $D>1$ denote $\mathcal{A}_{D}=\{1 / D<|y|<D\}$. Then, by the Harnack inequality of Serrin [9] we infer that for each $D>1$ there exist $K_{D}>1$, such that for any $r<R /(2 D)$ we have

$$
\frac{1}{K_{D}} \leq v_{r}(y) \leq K_{D} \quad \text { on } \mathcal{A}_{D} .
$$

Then, from the regularity results of [11, 4] it follows that there exist $L_{D}>0$ and $\alpha_{D} \in(0,1)$, such that for each $r<R /(2 D)$ we have

$$
\left\|v_{r}(y)\right\|_{C^{1, \alpha_{D}\left(\mathcal{A}_{D / 2}\right)}} \leq L_{D}
$$

where we denoted $\mathcal{A}_{D / 2}=\{2 / D<|y|<D / 2\}$. Set $Q:=\limsup _{r \rightarrow 0^{+}} \frac{\bar{u}(r)}{\underline{u}(r)}$ and choose a sequence $r_{n} \rightarrow 0$ such that $\lim _{n \rightarrow \infty} \frac{\bar{u}\left(r_{n}\right)}{\underline{u}\left(r_{n}\right)}=Q$. Using (2.15) we deduce from (2.13) 
that for a subsequence, still denoted by $\left\{r_{n}\right\}$, we have $v_{r_{n}} \rightarrow w_{D}$ in $C^{1}\left(\mathcal{A}_{D / 2}\right)$, where $w_{D}$ is a positive solution of

$$
-\Delta_{p} w_{D}=c_{p, N}^{*} \frac{w_{D}^{p-1}}{|y|^{p}} \quad \text { on } \mathcal{A}_{D / 2}
$$

Choosing a sequence $D_{m} \nearrow \infty$ and passing to a diagonal subsequence, we obtain a subsequence $r_{n_{k}} \rightarrow 0$ such that $v_{r_{n_{k}}} \rightarrow v_{0}$ in $C_{l o c}^{1}\left(\mathbb{R}^{N} \backslash\{0\}\right)$, where $v_{0}$ is a positive solution of (1.4). By Theorem 1.3 $v_{0}(x)=C|x|^{1-N / p}$, hence $Q=1$.

\section{UNIQUENESS OF THE EIGENFUNCTION IN THE CRITICAL CASE}

This section is devoted to the proof of Theorem 1.2. We shall need the following comparison lemma.

Lemma 3.1. Let $\Omega$ be a bounded domain in $\mathbb{R}^{N}$ of class $C^{2}$ and let $K \subset \subset \Omega$ be a compact subset. Suppose that $u_{1}, u_{2} \in C(\overline{\Omega \backslash K}) \cap W^{1, p}(\Omega \backslash K)$ are such that $u_{2}>0$ on $(\Omega \backslash K) \cup \partial K$ and $u_{1}=0$ on $\partial \Omega$. Suppose further that

$$
\begin{array}{ll}
-\Delta_{p} u_{1}-a(x)\left|u_{1}\right|^{p-2} u_{1} \leq 0 & \text { in } \Omega \backslash K, \\
-\Delta_{p} u_{2}-a(x)\left|u_{2}\right|^{p-2} u_{2} \geq 0 & \text { in } \Omega \backslash K,
\end{array}
$$

for some $a(x) \in L_{\text {loc }}^{\infty}(\Omega \backslash K)$, and that

$$
u_{2} \geq u_{1} \quad \text { on } \partial K \text {. }
$$

Then,

$$
u_{2}(x) \geq u_{1}(x) \quad \text { on } \Omega \backslash K .
$$

Proof. The result follows directly from the argument of Proposition A.1 and Corollary A.1 in [10]. The results in [10] deal with the case where $K=\{x \in \Omega, \operatorname{dist}(x, \partial \Omega)$ $\geq \beta\}$ for some $\beta>0$, but it is clear that the argument there works for general $K$.

Proof of Theorem 1.2. Let $u_{1}, u_{2}$ be two solutions to (1.1). Again by regularity theory we have $u_{1}, u_{2} \in C^{1}(\bar{\Omega} \backslash\{0\})$. For all sufficiently small $r>0$ set

$$
f(r)=\min _{\{|x|=r\}} \frac{u_{2}(x)}{u_{1}(x)} \quad, \quad F(r)=\max _{\{|x|=r\}} \frac{u_{2}(x)}{u_{1}(x)} .
$$

Thanks to Corollary 2.1 we have $\lim _{r \rightarrow 0^{+}} F(r) / f(r)=1$, and we can define

$$
\begin{aligned}
& m:=\liminf _{r \rightarrow 0^{+}} f(r)=\liminf _{r \rightarrow 0^{+}} F(r) \in[0, \infty], \\
& M:=\limsup _{r \rightarrow 0^{+}} f(r)=\limsup _{r \rightarrow 0^{+}} F(r) \in[0, \infty] .
\end{aligned}
$$

We can assume without loss of generality that $m<\infty$ (otherwise we interchange $u_{1}$ and $\left.u_{2}\right)$. For every $\varepsilon>0$ there exists a sequence $r_{n} \rightarrow 0$ such that

$$
u_{2}(x) \leq(m+\varepsilon) u_{1}(x) \quad \text { on }\left\{|x|=r_{n}\right\} .
$$

By Lemma 3.1 it follows that

$$
u_{2}(x) \leq(m+\varepsilon) u_{1}(x) \quad \text { on } \Omega \backslash B_{r_{n}}(0) .
$$

Letting $n$ tend to $\infty$ in (3.2) and then sending $\varepsilon$ to 0 , we obtain that

$$
u_{2}(x) \leq m u_{1}(x) \text { on } \Omega \backslash\{0\} .
$$

It follows that $M=m>0$. Interchanging $u_{1}$ and $u_{2}$ and repeating the above argument we also get that $u_{2}(x) \geq m u_{1}(x)$ and we conclude that $u_{2}=m u_{1}$. 
Remark 3.1. In the linear case $p=2$ the uniqueness (up to a multiplicative factor) of a solution to (1.1) is easy for any $\mu$ and $\lambda$. Indeed, if $u$ and $v$ are two solutions, we have by (4.12) below that $v \geq c_{1} u$ in $\Omega$ for some $c_{1}>0$. Defining $\bar{c}_{1}:=\sup \left\{c_{1}>\right.$ $\left.0 ; v \geq c_{1} u\right\}$ we have $v-\bar{c}_{1} u \geq 0$ in $\Omega$. If $v-\bar{c}_{1} u=0$, we are done. Otherwise, since $v-\bar{c}_{1} u$ is also a solution of the equation in (1.1) (recall that we are in the linear case), we have by the strong maximum principle $v-\bar{c}_{1} u>0$ in $\Omega$. Hence by the above argument there exists $c_{2}>0$ such that $v-\bar{c}_{1} u>c_{2} u$ in $\Omega$. This is a contradiction to the maximality of $\bar{c}_{1}$.

\section{Appendix: Existence And uniqueness in the SUbCRitical CASE}

This section is devoted to the subcritical case $I[\lambda]<c_{p, N}^{*}$. Existence of a minimizer in (1.2) is established in the next proposition.

Proposition 4.1. Assume that $I[\lambda]<c_{p, N}^{*}$. Let $\left\{u_{n}\right\} \subset W_{0}^{1, p}(\Omega)$ be a sequence of nonnegative functions satisfying

$$
\int_{\Omega} \frac{u_{n}^{p}}{|x|^{p}}=1, \forall n,
$$

and

$$
\lim _{n \rightarrow \infty} \int_{\Omega}\left|\nabla u_{n}\right|^{p}-\lambda \int_{\Omega} \frac{\eta\left|u_{n}\right|^{p}}{|x|^{p}}=I[\lambda] .
$$

Then $u_{n} \rightarrow u$ strongly in $W_{0}^{1, p}(\Omega \backslash\{0\})$, where $u$ is a minimizer for (1.2).

Proof. We shall first prove the convergence of a subsequence. It is clear from (4.1)(4.2) that $\left\{u_{n}\right\}$ is bounded in $W_{0}^{1, p}(\Omega \backslash\{0\})$, and passing to a subsequence we may assume that

$$
u_{n} \rightarrow u \text { weakly in } W_{0}^{1, p}(\Omega \backslash\{0\}) \text { and } u_{n} \rightarrow u \text { in } L^{p}(\Omega)
$$

for some nonnegative $u \in W_{0}^{1, p}(\Omega \backslash\{0\})$. We denote $v_{n}=u_{n}-u$. Fix any $r>0$ such that $B_{2 r}(0) \subset \Omega$. Then

$$
\left.\left|\int_{B_{2 r}(0)}\right| \nabla u_{n}\right|^{p}-\left|\nabla v_{n}\right|^{p} \mid \leq C\left\|\nabla\left(u_{n}-v_{n}\right)\right\|_{L^{p}\left(B_{2 r}(0)\right)}=C\|\nabla u\|_{L^{p}\left(B_{2 r}(0)\right)}:=\varepsilon_{r},
$$

with $\varepsilon_{r} \rightarrow 0$ as $r \rightarrow 0$. Let $\varphi_{r} \in C_{c}^{\infty}\left(\mathbb{R}^{N}\right)$ denote a cut-off function satisfying:

(i) $\varphi_{r}(x)=1$ for $|x| \leq r$,

(ii) $0 \leq \varphi_{r}(x) \leq 1$ for $r<|x|<2 r$,

(iii) $\varphi_{r}(x)=0$ for $|x| \geq 2 r$,

(iv) $\left\|\nabla \varphi_{r}\right\|_{L^{\infty}} \leq 4 / r$.

By Hölder's inequality,

$$
\begin{aligned}
\int_{B_{2 r}(0)}\left|\nabla\left(\varphi_{r} v_{n}\right)\right|^{p} \leq & \int_{B_{2 r}(0)}\left|\varphi_{r}\right|^{p}\left|\nabla v_{n}\right|^{p}+p \int_{B_{2 r}(0)}\left|v_{n} \nabla \varphi_{r}\right|\left(\left|v_{n} \nabla \varphi_{r}\right|+\left|\varphi_{r} \nabla v_{n}\right|\right)^{p-1} \\
\leq & \int_{B_{2 r}(0)}\left|\nabla v_{n}\right|^{p}+\frac{4 p}{r}\left(\int_{B_{2 r}(0)}\left(\left|v_{n} \nabla \varphi_{r}\right|+\left|\varphi_{r} \nabla v_{n}\right|\right)^{p}\right)^{\frac{p-1}{p}} \\
& \cdot\left(\int_{B_{2 r}(0)}\left|v_{n}\right|^{p}\right)^{\frac{1}{p}} .
\end{aligned}
$$


Therefore, using (4.3) we infer that

$$
\int_{B_{2 r}(0)}\left|\nabla\left(\varphi_{r} v_{n}\right)\right|^{p} \leq \int_{B_{2 r}(0)}\left|\nabla v_{n}\right|^{p}+D_{r} \alpha_{n},
$$

with $D_{r}>0$ and $\alpha_{n} \rightarrow 0$. By Hardy's inequality (1.3) we get

$$
\int_{B_{2 r}(0)}\left|\nabla\left(\varphi_{r} v_{n}\right)\right|^{p} \geq c_{p, N}^{*} \int_{B_{2 r}(0)} \frac{\left|\varphi_{r}\right|^{p}\left|v_{n}\right|^{p}}{|x|^{p}} \geq c_{p, N}^{*} \int_{B_{r}(0)} \frac{\left|v_{n}\right|^{p}}{|x|^{p}},
$$

which together with (4.4) and 4.5) yields

$$
\int_{B_{2 r(0)}}\left|\nabla u_{n}\right|^{p} \geq c_{p, N}^{*} \int_{B_{r}(0)} \frac{\left|v_{n}\right|^{p}}{|x|^{p}}-D_{r} \alpha_{n}-\varepsilon_{r}
$$

On the other hand, as in 4.4 we get

$$
\left|\int_{B_{r}(0)} \frac{u_{n}^{p}}{|x|^{p}}-\int_{B_{r}(0)} \frac{\left|v_{n}\right|^{p}}{|x|^{p}}\right| \leq C\left(\int_{B_{r}(0)}\left(\frac{u}{|x|}\right)^{p}\right)^{1 / p}:=\delta_{r},
$$

with $\delta_{r} \rightarrow 0$ as $r \rightarrow 0$ (by (1.3)). Moreover, by (4.3) and (1.3) we easily deduce that

$$
\lim _{n \rightarrow \infty} \int_{\Omega} \frac{\eta u_{n}^{p}}{|x|^{p}}-\int_{\Omega} \frac{\eta u^{p}}{|x|^{p}}=0 .
$$

By (4.1), (4.6) and (4.7) we obtain

$$
\begin{gathered}
\int_{\Omega}\left|\nabla u_{n}\right|^{p}-\lambda \int_{\Omega} \frac{\eta u_{n}^{p}}{|x|^{p}} \geq \int_{\Omega \backslash B_{2 r}(0)}\left|\nabla u_{n}\right|^{p}+c_{p, N}^{*}\left(1-\int_{\Omega \backslash B_{r}(0)} \frac{u_{n}^{p}}{|x|^{p}}\right)-\lambda \int_{\Omega} \frac{\eta u_{n}^{p}}{|x|^{p}} \\
-c_{p, N}^{*} \delta_{r}-D_{r} \alpha_{n}-\varepsilon_{r} .
\end{gathered}
$$

Passing to the limit $n \rightarrow \infty$ in (4.9) and using (4.2), (4.3) and (4.8), yields

$$
I[\lambda] \geq \int_{\Omega \backslash B_{2 r}(0)}|\nabla u|^{p}+c_{p, N}^{*}\left(1-\int_{\Omega \backslash B_{r}(0)} \frac{u^{p}}{|x|^{p}}\right)-\lambda \int_{\Omega} \frac{\eta u^{p}}{|x|^{p}}-c_{p, N}^{*} \delta_{r}-\varepsilon_{r} .
$$

Passing to the limit $r \rightarrow 0$ in (4.10) gives, using the definition of $I[\lambda]$,

$$
\begin{aligned}
I[\lambda] & \geq \int_{\Omega}|\nabla u|^{p}+c_{p, N}^{*}\left(1-\int_{\Omega} \frac{u^{p}}{|x|^{p}}\right)-\lambda \int_{\Omega} \frac{\eta u^{p}}{|x|^{p}} \\
& \geq c_{p, N}^{*}\left(1-\int_{\Omega} \frac{u^{p}}{|x|^{p}}\right)+I[\lambda] \int_{\Omega} \frac{u^{p}}{|x|^{p}} .
\end{aligned}
$$

By (4.11) and our assumption $I[\lambda]<c_{p, N}^{*}$ it follows that $\int_{\Omega} \frac{u^{p}}{|x|^{p}}=1$, and then by (4.11) we infer that $u$ is a minimizer. Since $\int_{\Omega} \frac{u_{n}^{p}}{|x|^{p}} \rightarrow \int_{\Omega} \frac{u^{p}}{|x|^{p}}=1$ we also get that $\int_{\Omega}\left|\nabla u_{n}\right|^{p} \rightarrow \int_{\Omega}|\nabla u|^{p}$, and the strong convergence $u_{n} \rightarrow u$ in $W_{0}^{1, p}(\Omega \backslash\{0\})$ follows. So far we proved the convergence of a subsequence of $\left\{u_{n}\right\}$. But since it is known that a solution to (1.1) in $W_{0}^{1, p}(\Omega \backslash\{0\})$ is unique up to a multiplicative factor (see [1, Theorem 2.1] and [7] Proposition 3.2]) the full convergence $u_{n} \rightarrow u$ follows as well. 
Proof of Theorem 1.1. Existence of a positive minimizer $u$ for (1.2), which is then a solution of (1.1), follows directly from Proposition 4.1. Next we turn to the proof of the uniqueness part. Let $v$ be another solution to (1.1) with $\mu=I[\lambda]$. We claim that there exists $c_{1}>1$ such that

$$
\frac{u}{c_{1}} \leq v \leq c_{1} u \quad \text { on } \Omega \backslash\{0\} .
$$

Indeed, to see for example why $u / v$ is bounded, assume by negation that $\sup _{\Omega} u / v=$ $\infty$. By Hopf's boundary lemma [12] we must have $\lim _{r \rightarrow 0} \sup _{B_{r}(0) \backslash\{0\}} u / v=\infty$. Using Harnack's inequality [9] we obtain for some sequence $r_{n} \rightarrow 0$ that $u \geq n v$ on $\partial B_{r_{n}}(0)$. But then we get by Lemma 3.1 that $u \geq n v$ on $\Omega \backslash B_{r_{n}}(0)$. This is clearly a contradiction for $n$ large enough, and (4.12) follows. On the other hand, by a simple rescaling argument, Harnack inequality $([9])$ and $C^{1}$ regularity estimates ([11, 4]), it follows (see [10, Lemma A.3]) that, for some $c_{2}>0$, we have

$$
\left|\left(\frac{\nabla v}{v}\right)(x)\right| \leq \frac{c_{2}}{|x|} \quad \text { on } B_{d}(0) \backslash\{0\}, \text { with } d:=\frac{1}{2} \min \{|y| ; y \in \partial \Omega\} .
$$

Combining (4.12) and (4.13) we get that

$$
|\nabla v(x)| \leq c_{1} c_{2} \frac{u(x)}{|x|} \quad \text { on } B_{d}(0) \backslash\{0\} .
$$

By (4.14) and (1.3) we obtain that $v \in W_{0}^{1, p}(\Omega \backslash\{0\})$ and therefore $v$ is a minimizer for (1.2). The fact that $v$ is a multiple of $u$ follows from the argument cited at the end of the proof of Proposition 4.1, To complete the proof we remark that in 8 it was proved that there is no solution in $W_{0}^{1, p}(\Omega \backslash\{0\})$ to (1.1) with $\mu=c_{p, N}^{*}$.

\section{ACKNOWLEDGements}

The authors are grateful to Professor Yehuda Pinchover for many interesting discussions.

\section{REFERENCES}

[1] W. Allegretto and Y. X. Huang, A Picone's identity for the $p$-Laplacian and applications, Nonlinear Analysis TMA 32 (1998), 819-830. MR1618334 (99c:35051)

[2] A. Anane, Simplicité et isolation de la première valeur propre du p-laplacien avec poids, C. R. Acad. Sci. Paris 305, Série I (1987), 725-728. MR0920052 (89e:35124)

[3] H. Brezis and M. Marcus, Hardy's inequality revisited, Ann. Sc. Norm. Pisa. 25 (1997), 217-237. MR 1655516 (99m:46075)

[4] E. Di Benedetto, $C^{1+\alpha}$ local regularity of weak solutions of degenerate elliptic equations, Nonlinear Anal. TMA 7 (1983), 827-850. MR0709038 (85d:35037)

[5] P. L. Lions, The concentration-compactness principle in the calculus of variations. The limit case. I., Rev. Mat. Iberoamericana 1 (1985), 145-201. MR0834360 (87c:49007)

[6] M. Marcus, V. J. Mizel and Y. Pinchover, On the best constant for Hardy's inequality in $\mathbb{R}^{n}$, Trans. A.M.S. 350 (1998), 3237-3255. MR.1458330 (98k:26035)

[7] M. Marcus and I. Shafrir, An eigenvalue problem related to Hardy's $L^{p}$ inequality, Ann. Sc. Norm. Pisa. 29 (2000), 581-604. MR1817710 (2002c:49082)

[8] A. Poliakovsky, On minimization problems which approximate Hardy's $L^{p}$ inequality, Nonlinear Anal. 54 (2003), 1221-1240. MR.1995927|(2004h:42024)

[9] J. Serrin, Local behavior of solutions of quasi-linear equations, Acta Math. 111 (1964), 247302. MR0170096 (30:337)

[10] I. Shafrir, Asymptotic behavior of minimizing sequences for Hardy's inequality, Commun. Contemp. Math. 2 (2000), 151-189. MR1759788 (2001f:35163) 
[11] P. Tolksdorf, Regularity for a more general class of quasilinear elliptic equations, J. Diff. Eqns. 51 (1984), 121-150. MR0727034 (85g:35047)

[12] J. L. Vázquez, A strong Maximum Principle for Some Quasilinear Elliptic Equations, Appl. Math. Optim. 12 (1984), no. 3, 191-202. MR0768629 (86m:35018)

Department of Mathematics, Technion - Israel Institute of Technology, 32000 Haifa, ISRAEL

E-mail address: maarkady@tx.technion.ac.il

Department of Mathematics, Technion - Israel Institute of Technology, 32000 Haifa, ISRAEL

E-mail address: shafrir@tx.technion.ac.il 